\title{
RENDIMENTO DE ÓLEO ESSENCIAL DE Corymbia citriodora HILL \& JOHNSON SOB DIFERENTES NÍVEIS DE INFESTAÇÃO DE GALHAS DE Epichrysocharis burwelli SCHAUFF (HYMENOPTERA, EULOPHIDAE)
}

\author{
Rosylaine Aparecida Pereira ${ }^{1}$, Evoneo Berti Filho ${ }^{2}$, Rogerio Goularte Moura ${ }^{3}$ \\ ${ }^{1}$ Depto. Produção Vegetal-FAC/UNESP, Lageado, CEP: 18.610-307, Botucatu, SP, e-mail: rosylaine@ gmail.com \\ ${ }^{2}$ Depto. Entomologia e Acarologia - ESALQ/USP - Caixa postal 9, CEP 13.418-900, Piracicaba, SP, e-mail: eberti@ usp.br \\ ${ }^{3}$ Doutorando em Ecologia Aplicada-CENA/USP, e-mail: rgmoura@ gmail.com
}

\section{RESUMO}

O objetivo do trabalho foi avaliar a influência de diferentes níveis de infestação de galhas de Epichrysocharis burwelli (Hymenoptera, Eulophidae) sobre o rendimento de óleo essencial de folhas de Corymbia citriodora. Foram tomadas 10 plantas de C. citriodora ao acaso, e em cada uma delas 16 folhas do terço médio foram coletadas (quatro em cada quadrante da planta), totalizando 160. Cada folha teve sua área medida e as galhas contadas, para a obtenção do número de galhas $/ \mathrm{cm}^{2}$. Além disso, 250 galhas coletadas ao acaso, de folhas e árvores diferentes, tiveram sua área medida, para verificar a porcentagem de área foliar ocupada. Através desses dados, foram estabelecidos três níveis de infestação: baixo $(0,04-18,72 \%$ da área foliar ocupada com galhas), médio (18,76-37,88\% da área ocupada) e alto (37,92-57,32\% da área ocupada). Para cada um desses níveis o rendimento de óleo essencial foi avaliado, através de hidrodestilação, e comparado com o rendimento de folhas sem galhas. Verificou-se que a produção de óleo foi influenciada pelo nível de infestação das galhas, onde altos níveis de galhas em folhas causaram efeito significativamente negativo sobre o rendimento de óleo essencial, que ainda pode ser potencializado devido à queda prematura de folhas, que ocorre quando estas estão sob altas infestações.

Palavras-chave: Vespa-da-galha, danos, eucaliptos, Brasil

\section{ABSTRACT}

This paper evaluates the yield of essencial oil from leaves of Corymbia (=Eucalyptus) citriodora under different levels of infestation of galls of Epichrysocharis burwelli Schauff (Hymenoptera, Eulophidae). Ten E. citriodora plants were randomly taken and from each one 16 leaves were collected. The area of each leaf was measured and the galls were counted thus obtaining the number of galls per square centimeter. Also the areas of 250 galls randomly collected from different leaves and trees were measured in order to set the average percentage of leaf are occupied by them. According to the percentage of leaf area occupied by the galls three levels of infestation were set: low $(0.04-18.78 \%)$, medium (18.76 - 37.88\%) and high (37.92 $57.32 \%$ ). By hydrodestilation of the leaves the essential oil yield was evaluated for each level. It was observed that oil yield was influenced by the level of gall infestation, with high levels of leaf galls causing a significantly negative effect on essential oil yield, which may also be potentialized by the falling of leaves from eucalypt trees under high gall infestation.

Keywords: Gall wasp, damages, eucalypts, Brazil 


\section{INTRODUÇÃO}

No Brasil são cultivadas várias espécies de plantas aromáticas produtoras de óleo essencial, dentre as quais, as espécies dos gêneros Eucalyptus e Corymbia, conhecidas vulgarmente como eucaliptos, destacam-se pela excelente qualidade do óleo produzido e pela facilidade de produção (Vitti \& Brito, 1999; Andrade \& Gomes, 2000).

Corymbia citriodora, até recentemente conhecida como Eucalyptus citriodora (Hill \& Johnson, 1995), é considerada a espécie de maior produção de óleo essencial no mundo, sendo bastante disseminada no Brasil, principalmente em pequenas e médias propriedades rurais, apresentando múltiplas finalidades, como matéria-prima para construção rural, para cercas e postes e para a produção de carvão e lenha. No entanto, nenhum destes fins apresenta-se tão viável financeira $\mathrm{e}$ operacionalmente como a extração de óleo essencial (Vitti \& Brito, 1999).

A produção e qualidade do óleo essencial podem ser influenciadas por fatores genéticos e fisiológicos da planta, pelas técnicas de extração utilizadas e também por fatores ambientais, bióticos e abióticos. Dentre os fatores bióticos, a ocorrência de pragas pode ser um fator limitante ao cultivo desta essência, dependendo da densidade populacional em que estas se encontram (Boland et al., 1991).

C. citriodora é tida como uma das espécies de eucalipto mais atacadas por insetos, sendo os maiores danos ocasionados por representantes das ordens Hymenoptera (formigas cortadeiras), Lepidoptera (lagartas desfolhadoras) e Coleoptera (besouros desfolhadores) (Berti Filho, 1984). Além destas, uma nova praga recém-introduzida, Epichrysocharis burwelli (Hymenoptera: Eulophidae: Tetrastichinae), vem ocasionando danos ao cultivo de $C$. citriodora e, por isso, foi recentemente incluída no grupo de pragas importantes para esta cultura (Berti Filho, 2003; Anjos \& Zacaro, 2005).

E. burwelli, também conhecida como "vespa-da-galha", é uma microvespa $(0,5-$ $0,6 \mathrm{~mm}$ de comprimento) originária da Austrália, que induz galhas lenticulares no limbo foliar de plantas de $C$. citriodora, em densidades bastante elevadas (LaSalle, 2005). No ano de 2000 foi registrada pela primeira vez, na cidade de Los Angeles (EUA) (Shauff \& Garrison, 2000) e em 2003 foi encontrada no Brasil, no estado de Minas Gerais (Berti Filho, 2003). Atualmente encontra-se amplamente distribuída em vários estados brasileiros, principalmente naqueles da região sul e sudeste (Anjos \& Zacaro, 2005).

Após o aparecimento dessa nova praga nos plantios de C. citriodora, as destilarias verificaram uma redução significativa no rendimento de óleo essencial (cerca de 50\%), que ocorreu provavelmente devido às galhas induzidas na epiderme foliar, que em altas infestações provocam um necrosamento na folha (Anjos \& Zacaro, 2005). Apesar dessa drástica redução na quantidade de óleo essencial extraído observada pelas destilarias, nenhum estudo ainda havia sido realizado para verificar o real efeito da indução de galhas de $E$. burwelli sobre a produção de óleo essencial de sua planta hospedeira. Na tentativa de minimizar essa carência, essa pesquisa teve como objetivos, definir níveis de infestação de galhas de acordo com a porcentagem de área foliar ocupada por estas e, para cada um dos níveis, verificar a influência sobre o rendimento de óleo essencial de Corymbia citriodora.

\section{MATERIAL E MÉTODOS}

Para verificar a influência das galhas de E. burwelli na produção de óleo essencial de $C$. citriodora, foram conduzidos dois experimentos distintos. No primeiro, foram 
atribuídos níveis de infestação, verificandose a porcentagem de área ocupada pelas galhas em relação à área da folha. No segundo experimento, os níveis de infestação atribuídos foram utilizados para a coleta de amostras e a avaliação do rendimento de óleo para cada um deles.

\section{Experimento I}

Foram realizadas coletas de folhas durante o mês de janeiro de 2009, em um talhão de 0,8 ha de $C$. citriodora, pertencente à Estação Experimental de Estudos Florestais (ESALQ/USP), localizada no município de Itatinga/SP, nas seguintes coordenadas geográficas: latitude de $23^{\circ} 10^{\prime} \mathrm{S}$; longitude de $48^{\circ} 40^{\prime} \mathrm{W}$ e altitude de $850 \mathrm{~m}$. O clima da região, segundo a classificação de Köeppen, é do tipo Cwa Tropical Úmido (com chuvas de verão e três meses mais secos: junho, julho e agosto). A precipitação e temperatura média anual são de $1350 \mathrm{~mm}$ e $20^{\circ} \mathrm{C}$, respectivamente.

Foram realizadas duas coletas de folhas, com intervalo de quinze dias entre elas. Em cada coleta, cinco plantas foram tomadas ao acaso dentro do talhão, e nestas somente folhas do terço médio e de mesma idade foram coletadas (folhas totalmente desenvolvidas). Em cada uma das árvores foram retiradas 16 folhas, que foram acondicionadas separadamente em sacos plásticos devidamente rotulados, imediatamente após a coleta. Ao todo, 160 folhas foram coletadas.

Após a coleta, o material foi levado ao laboratório e armazenado em câmara fria até o momento das análises. A área foliar foi obtida através da digitalização da imagem de cada folha com o auxílio de um "scanner", e posteriormente o programa SIARC (versão 3.0), foi utilizado para calcular a área em $\mathrm{cm}^{2}$. As galhas presentes em cada folha foram contadas, nas superfícies adaxial e abaxial, obtendo-se assim o total de galhas $/ \mathrm{cm}^{2}$.
A área média $\left(\mathrm{cm}^{2}\right)$ ocupada pelas galhas foi obtida selecionando-se cinco folhas/árvore, de cinco árvores diferentes. Para cada uma delas, dez galhas foram medidas utilizando-se o programa LEICA APPLICATION SUITE (versão 3.4.1). Com esses resultados foram definidos três níveis de infestação: baixo, médio e alto.

\section{Experimento II}

Os três níveis de infestação, definidos no experimento anterior, foram utilizados para a coleta de folhas, que ocorreu na primeira semana de abril de 2009, na mesma área já mencionada, em plantas conduzidas em sistema de rebrota com ramos de aproximadamente um ano de idade. Para cada um dos níveis definidos, o rendimento de óleo essencial foi avaliado e comparado com aquele de folhas sem a presença de galhas.

Foram coletadas amostras de $1,5 \mathrm{Kg}$ de folhas para cada um dos tratamentos e para a testemunha, as quais foram embaladas em sacos plásticos escuros imediatamente após a coleta, e armazenadas em câmara fria, regulada para $-20^{\circ} \mathrm{C}$, até o momento das análises, que ocorreram em menos de 24 horas após a coleta.

O rendimento de óleo foi verificado através de hidrodestilação das folhas durante 50 minutos. Para cada tratamento e testemunha, foram utilizadas cinco repetições, com $150 \mathrm{~g}$ de folhas para cada uma delas, com duas repetições. O óleo coletado foi pesado em balança analítica para posterior cálculo do rendimento. Foi realizada uma correção de peso para volume utilizando-se a densidade do óleo (aproximadamente 0,86). A cada hidrodestilação toda a vidraria foi limpa com álcool $92 \%$ e água corrente.

Os rendimentos de óleo para cada um dos níveis de infestação e da testemunha foram comparados através de análise de variância e as médias foram separadas 
através do teste de Tukey, ao nível de $1 \%$ de probabilidade. A presença de galhas nas superfícies adaxial e abaxial foi comparada através do Teste- $t$. O programa SAS foi utilizado.

\section{RESULTADOS E DISCUSSÃO}

As galhas de Epichrysocharis burwelli são pequenas, lenticulares e expressas em ambas as superfícies foliares (Tabela 1), tendo sido encontradas em 65,4\% das folhas avaliadas, com densidades bastante variáveis, entre 0,04 a 44,10 galhas $/ \mathrm{cm}^{2}$. Em folhas mais infestadas foram contadas até 1400 galhas.

Em relação à distribuição da densidade de galhas nas folhas verificou-se maior frequiência das densidades menores $(0-$ 100 galhas/folha, representando 63\% do total). Em 55 folhas $(34,38 \%$ do total) não foram encontradas galhas e os níveis mais altos de densidade ocorreram com frequiências bastante baixas nas folhas avaliadas.

De acordo com as densidades de galhas verificadas, as infestações foram divididas em quatro classes: sem infestação, infestação baixa (1-468 galhas/folha), infestação média (469-947 galhas/folha) e infestação alta (948-1433 galhas/folha). Como a área média de folhas observada foi de $29,28 \mathrm{~cm}^{2}$, e das galhas de $0,04 \mathrm{~cm}^{2}$, as infestações, baixa, média e alta, tiveram em média uma perda em área ocasionada pelas galhas de 9,34\% (0,04-18,72\%), 28,32\% $(18,76-37,88 \%)$ e $47,62 \%(37,92-57,32 \%)$, respectivamente.

Analisando os resultados de rendimento em óleo em relação aos níveis de infestação de galhas em folhas obtidos no primeiro experimento, verificou-se que a presença de galhas nas folhas afeta significativamente o rendimento de óleo de C. citriodora. As plantas que não apresentavam galhas e aquelas com níveis médios de ataque apresentaram as maiores médias de rendimento, diferindo significativamente dos outros dois tratamentos (baixo e alto) $(\mathrm{F}=4,93 ; \mathrm{P}<0,005)$ (Figura 1).

Os resultados obtidos neste estudo são coerentes aos observados na literatura para a mesma espécie de galhador e para outras espécies da subfamília Tetrastichinae que também induzem galhas em folhas de eucaliptos. LaSalle (2005) cita densidades de galhas de $E$. burwelli em folhas de $C$. citriodora, sob alta infestação, superiores a 40 galhas $/ \mathrm{cm}^{2}$. Protasov et al. (2007), avaliando a correlação entre densidade $\mathrm{e}$ tamanho das galhas de Ophelimus eucalypti em folhas de Eucalyptus camaldulensis, verificaram um claro relacionamento entre esses dois parâmetros e observaram densidades entre 11,5 a 36,0 galhas $/ \mathrm{cm}^{2}$, com diâmetros das galhas variando entre 1,2 a $0,9 \mathrm{~mm}$, respectivamente. Withers et al. (2000) encontraram diferenças entre o número médio de galhas dessa mesma espécie de galhador em folhas dependendo da espécie de eucalipto avaliada; em $E$. botryoides, E. saligna e E. deanei os números médios de galhas nas folhas infestadas foram de 10,7, 11,7 e 1,8, respectivamente, indicando uma diferença na densidade de galhas dependendo da suscetibilidade da espécie de hospedeiro utilizada.

O rendimento em óleo essencial é considerado uma das mais importantes características a ser avaliada nos plantios destinados à produção comercial, expressando a quantidade de óleo produzido a partir de uma determinada quantia de 
Tabela 1. Número médio de galhas de Epichrysocharis burwelli encontrado nas superfícies adaxial e abaxial de folhas de Corymbia citriodora coletadas na Estação Experimental de Estudos Florestais de Itatinga/SP.

\begin{tabular}{ccc}
\hline Posição na folha & Superfície da folha & Número médio de galhas \\
\hline & Adaxial & $164,34 \mathbf{a}$ \\
Proximal & Abaxial & $166,90 \mathbf{a}$ \\
\hline & Adaxial & $182,97 \mathbf{a}$ \\
Mediana & Abaxial & $180,31 \mathbf{a}$ \\
\hline & Adaxial & $60,12 \mathbf{a}$ \\
Distal & Abaxial & $61,12 \mathbf{a}$ \\
\hline
\end{tabular}

Médias seguidas da mesma letra na coluna não diferem entre si, pelo Teste- $t$, ao nível de $1 \%$ de probabilidade.

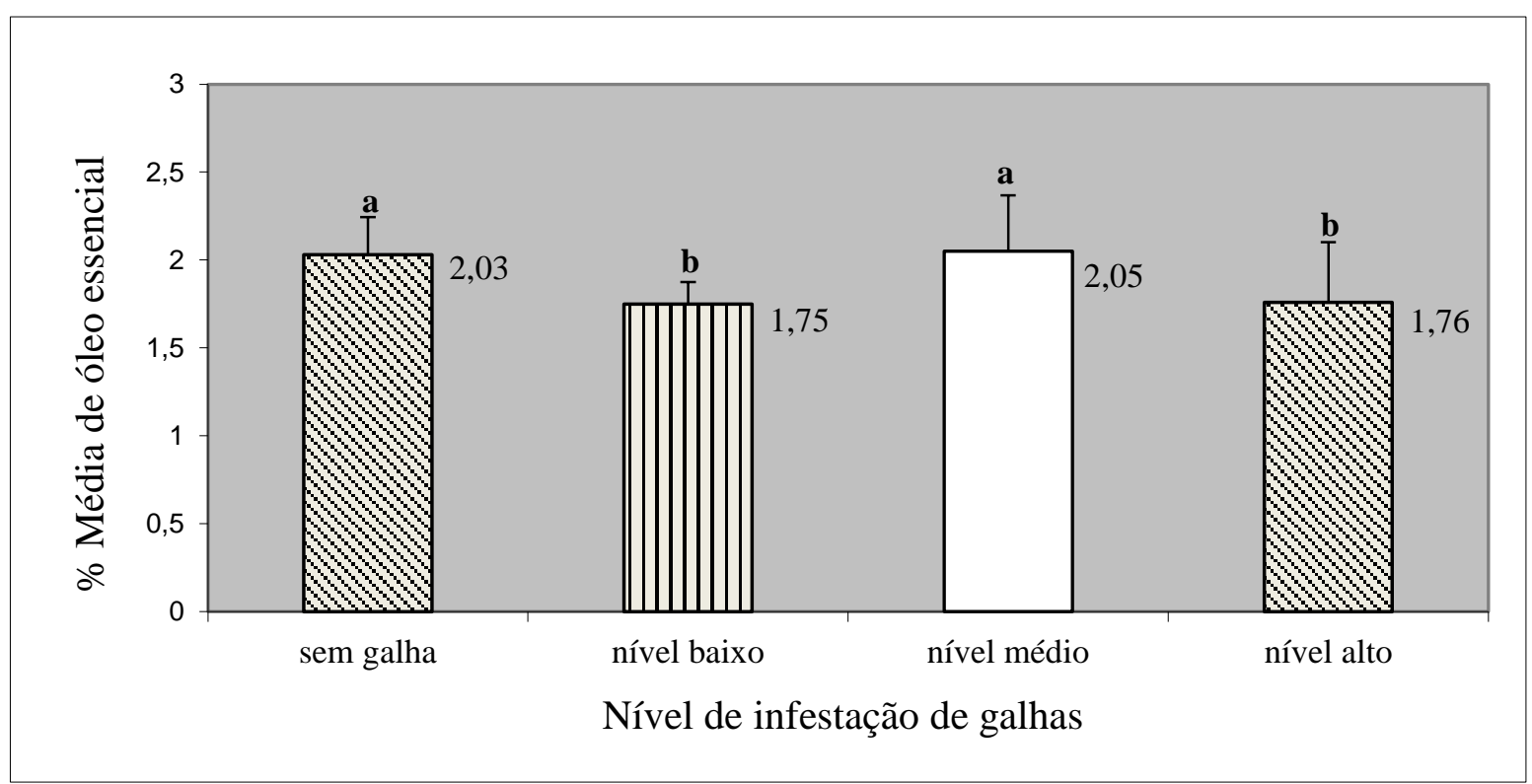

Figura 1. Porcentagem média de óleo essencial extraído de folhas de C. citriodora, coletadas no Horto Florestal de Itatinga/SP em diferentes níveis de infestação de galhas de E. burwelli. Médias de mesma letra não diferem entre si pelo Teste de Tukey $(\mathrm{P}<0,005)$.

folha. Em termos médios, no Brasil, os plantios comerciais de $C$. citriodora destinados à extração de óleo essencial apresentam rendimentos variando de 1,2 a 1,4\% (Vitti \& Brito, 1999). A produtividade, a composição e a quantidade dos constituintes dos óleos essências são bastante influenciadas por vários fatores ambientais, como condições do solo, umidade relativa, radiação solar, temperatura e o estresse hídrico, e por outros fatores como parte da planta utilizada, sua idade, o tipo de manejo florestal, o método de amostragem da folha, o processo de extração e de análise do óleo, além da variabilidade genética (Vitti \& Brito, 2003). Qualquer um desses fatores ou mais de um deles podem ter determinado variações no rendimento de 
óleo essencial extraído, que não provocadas pelas galhas em si. No entanto, existe uma coerência no resultado quando se consideram as folhas sem galhas e aquelas com níveis altos de infestação, que apresentaram diferenças significativas entre si. Nos tratamentos intermediários podem ter ocorrido erros de coleta, justificando tais diferenças, já que é bastante difícil em condições de campo separar os níveis de infestação de galha, uma vez que são utilizadas quantidades relativamente grandes de folhas na extração.

A origem biossintética dos óleos essenciais de eucalipto relaciona-se com seu metabolismo secundário, que confere às plantas a capacidade de adaptação às condições do meio ambiente em que vivem, sendo que sua ocorrência estaria relacionada com a defesa da planta contra insetos, resistência ao frio quando no estágio de plântula e à redução da perda de água (Boland et al., 1991). No caso de espécies galhadoras, entretanto, essa ocorrência de compostos secundários e óleos, beneficiam o galhador por protegê-lo de insetos mastigadores, fungos e outros microorganismos (Blanche \& Westoby, 1995). A larva do inseto indutor de galha interfere nos tecidos da planta ao redor dela, mudando a alocação de nutrientes e compostos secundários. Os nutrientes são concentrados nos tecidos meristemáticos em contato com a larva, enquanto os compostos secundários, primariamente taninos, são transportados para os tecidos externos, que criam uma barreira química ao redor da galha (Fernandes \& Price, 1991). Portanto, mesmo havendo diminuição no rendimento de óleo em folhas com altos níveis de infestação, essa influência pode ser subestimada, uma vez que o inseto poderia preferir plantas com maiores teores de óleo em suas folhas.

Apesar de altas infestações de galhas em folhas terem reduzido significativamente a porcentagem de óleo essencial neste experimento, esse resultado talvez não corresponda à imensa diminuição verificada na produção das destilarias, após a entrada do inseto nas áreas de $C$. citriodora, chegando, segundo Anjos \& Zacaro (2005) a cerca de $50 \%$ em alguns locais. Possivelmente, o fator responsável por essa queda na produção seja o intenso desfolhamento observado em árvores sob altas infestações de E. burwelli. Durante a coleta de folhas para a extração de óleo essencial, houve uma grande dificuldade em se obter o peso necessário de folhas naquelas plantas altamente infestadas. Essas plantas geralmente eram menores e continham poucas folhas. Fernandes et al. (1999) avaliaram o efeito de galhas induzidas por Melaphis rhois (Aphididae) sobre a abscisão foliar de Rhus glabra (Anacardiaceae) e verificaram que aquelas plantas com galhas apresentavam uma queda de folhas mais intensa que aquelas que não continham galhas e que $o$ inseto tinha potencial influência sobre o desempenho da planta hospedeira, reduzindo a área foliar e fotossíntese em indivíduos suscetíveis.

\section{CONCLUSÃO}

Com base nos resultados obtidos nos dois experimentos realizados, pode-se afirmar que a densidade média de galhas de Epichrysocharis burwelli em folhas de Corymbia citriodora é de sete galhas $/ \mathrm{cm}^{2}$, em plantio localizado na Estação Experimental de Estudos Florestais de Itatinga/SP, no entanto, existem folhas com densidades superiores a 40 gallhas $/ \mathrm{cm}^{2}$; as galhas visualizadas nas superfícies abaxial e adaxial são as mesmas e intensidades altas de galhas de E. burwelli nas folhas reduzem o rendimento de óleo essencial de $C$. citriodora. 


\section{AGRADECIMENTOS}

Ao Prof. Dr. José Otávio Brito, por ceder o laboratório para as análises de rendimento de óleo essencial; ao engenheiro florestal Rildo Moreira Moreira, pelo apoio dado nas coletas de campo e à CAPES (Coordenação de Aperfeiçoamento de Pessoal de Nível Superior), pela bolsa de estudos concedida.

\section{REFERÊNCIAS BIBLIOGRÁFICAS}

ANDRADE, A. M.; GOMES, S.S. 2000. Influência de alguns fatores não genéticos sobre o teor de óleo essencial em folhas de Eucalyptus citriodora Hook. Floresta e Ambiente, v. 7, p. 181-189.

ANJOS, N.; ZACARO, A. A. 2005. A microvespa Epichrysocharis burwelli Schauff (Hym.: Eulophidae): novíssima praga florestal no Brasil. In: Zanuncio, J.C., Pereira, J.M.M., Zanuncio, T.V. (Eds). Plagas Forestales Neotropicales, Manejo Integrado de Plagas y Agroecologia, n. 75, p. 90-92.

BERTI FILHO, E. 1984. O parasitismo no controle integrado de pragas florestais. Silvicultura, v. 10, p. 7-10.

BERTI FILHO, E.; COSTA, V. A.; LASALLE, J. 2003. Primeiro registro da vespa-da-galha, Epichrysocharis burwelli (Hymenoptera: Eulophidae) em Corymbia (Eucalyptus) citriodora (Myrtaceae) no Brasil. Revista de Agricultura, v. 79, p. 363-363.

BLANCHE, K. R.; WESTOBY, M. 1995. Gall-forming insect diversity is linked to soil fertility via host plant taxon. Ecology, v. 76, p. 2334-2337.

BOLAND, D. J.; BROPHY, J. J.; HOUSE, A.P.N. 1991. Eucalyptus leaf oils - use, chemistry, distillation and marketing. Melbourne: INKATA; ACIAR; CSIRO, 274pp.

FERNANDES, G. W.; CASTRO, F. M. C.; MARQUES, E. S. A. 1999. Leaflet abscission caused by a gall induced by Melaphis rhois (Homoptera: Aphididae) on Rhus glabra (Anacardiaceae). International Journal of Ecology and Environmental Sciences, v. 25, p. 63-69. HILL, K. D.; JOHNSON, L. A. S. 1995. Systematic studies in the eucalypts 7. A revision of the bloodwoods, genus Corymbia (Myrtaceae). Telopea, v. 6, p. 185-504.

LASALLE, J. 2005. Biology of gall inducers and evolution of gall induction in Chalcidoidea (Hymenoptera: Eulophidae, Eurytomidae, Pteromalidae, Tanaostigmatidae, Torymidae). In: Raman, A., Schaeffer, C.W., Withers, T.M. (Eds). Biology, ecology and evolution of gall-inducting arthropods. New Hampshire: Science Publishers, Inc., cap. 18, v. 2, p. 507-537.

PROTASOV, A.; LASALLE, J.; BLUMBERG, D.; BRAND, D.; SAPHIR, N.; ASSAEL, F.; FISHER, N., MENDEL, Z. 2007. Biology, revised taxonomy and impact on host plants of Ophelimus maskelli, an invasive gall inducer on Eucalyptus spp. in the Mediterranean Area. Phytoparasitica, v. 35, p. 50-76.

SCHAUFF, M. E.; GARRISON, R. 2000. An introduced species of Epichrysocharis (Hymenoptera: Eulophidae) producing galls on Eucalyptus in California with notes on the described species and placement of the genus. Journal of Hymenopteran Research, v. 9, p. 176181.

VITTI, A. M. S.; BRITO, J. O. 1999. Avaliação do rendimento e do teor de citronelal do óleo essencial de procedências e raças locais de Eucalyptus citriodora. Scientia Forestalis, v. 56, p. 145-154.

VITTI, A. M. S.; BRITO, O. J. 2003. Óleo essencial de eucalipto. Piracicaba: ESALQ. Documentos florestais $\mathrm{n}^{\circ} 17$, $\mathrm{p}$. 1-26. 
Rendimento de óleo essencial de Corymbia citriodora Hill \& Johnson sob diferentes níveis de infestação de galhas de Epichrysocharis burwelli Schauff (Hymenoptera, Eulophidae)

WITHERS, T. M.; RAMAN, A.; BERRY, J.A. 2000. Host range and biology of Ophelimus eucalypti (Gahan) (Hym., Eulophidae), a pest of New Zealand eucalypts. New Zealand Plant Protection, v. 53, p. 339-344. 